\title{
Overexpression of syntenin enhances hepatoma cell proliferation and invasion: Potential roles in human hepatoma
}

\author{
XUEMIN LIU*, XUFENG ZHANG* ${ }^{*}$ YI LV, JUNXI XIANG and JIANHUA SHI \\ Department of Hepatobiliary Surgery, The First Affiliated Hospital of Medical College, \\ Xi'an Jiaotong University, Xi'an, Shaanxi 710004, P.R. China
}

Received May 13, 2014; Accepted August 12, 2014

DOI: $10.3892 /$ or.2014.3498

\begin{abstract}
Hepatocellular carcinoma (HCC) ranks as the third leading cause of tumor-related mortality worldwide. Recently, syntenin was found to be upregulated in several tumors and to exert pivotal roles in the development of cancer. However, its function and the underlying mechanism in $\mathrm{HCC}$ remain to be defined. In the present study, the elevated expression levels of syntenin mRNA and protein were detected in four HCC cell lines. Overexpression of syntenin in hepatoma HCCLM3 cells enhanced cell proliferation. Furthermore, syntenin upregulation increased epidermal growth factor receptor (EGFR) expression, which accounted for syntenin-induced cell proliferation as precondition with EGFR siRNA clearly attenuated cell proliferation in syntenin-transfected cells. At the same time, syntenin overexpression promoted cell invasion by MMP-2, as pretreatment with anti-MMP-2 antibody blocked syntenin-induced invading cell numbers. Additionally, syntenin upregulation induced the phosphorylation of p38 MAPK contributing to the increase in MMP-2 expression, as treatment with the specific inhibitor for p38 MAPK (SB203580) clearly abrogated MMP-2 expression induced by syntenin. Collectively, our results suggest that syntenin overexpression plays a critical role in promoting the proliferation and invasion of hepatoma cells. Therefore, the present study provides new insight into how syntenin accelerates the development and progression of hepatoma, and suggests that syntenin may be a promising therapeutic agent against hepatoma.
\end{abstract}

\section{Introduction}

Hepatocellular carcinoma (HCC) ranks as the most common type of primary liver cancer with poor survival rates, and accounts for $90 \%$ of all liver cancers $(1,2)$. Its incidence is currently increasing around the world, particularly in Asia

Correspondence to: Dr Xuemin Liu, Department of Hepatobiliary Surgery, The First Affiliated Hospital of Medical College, Xi'an Jiaotong University, Xi'an, Shaanxi 710004, P.R. China

E-mail: xueminliuxian@163.com

*Contributed equally

Key words: hepatocellular carcinoma, syntenin, proliferation, invasion, EGFR, p38 MAPK and sub-Saharan Africa, with as many as 120 cases per $100,000(3,4)$. The high proliferation and invasion characteristics of HCC make it the leading cause of cancer-related mortality worldwide. Although considerable advances in the treatment of $\mathrm{HCC}$ have been made, its intrinsic resistance to many clinical therapies, such as chemotherapy and radiotherapy, have led to ineffective therapeutic options, and the 5 -year survival rate of patients is only $\sim 10 \%$ (5). Therefore, it is necessary to understand the molecular mechanism underlying HCC development and progression.

Syntenin, also known as melanoma differentiation associated gene-9 (mda-9), is an evolutionarily conserved sytosolic protein, that was initially identified as a molecule linking syndecan-mediated signaling to the cytoskeleton. It is now recognized as a significant member of the expanding family of scaffolding proteins with highly potent and diverse biological activities, including protein trafficking, cell adhesion, regeneration and signal transduction (6-8). Syntenin comprises four separate structural domains; the presence of two tandem PDZ domains (PDZ1 and PDZ2) is the notable feature, reflecting its indispensable role in assembling cell signaling processes. The function of syntenin has attracted increasing attention regarding its critical roles in cancer growth, invasion and metastasis (9-11). Extensive studies have demonstrated that syntenin is upregulated in several types of cancer, including breast cancer, melanoma and bladder cancer $(10,12,13)$. Overexpression of syntenin in breast cancer cells promotes cell migration, invasion and tumor growth, as well as poor patient survival, indicating a significant role in breast cancer progression (10). Blocking syntenin expression inhibits melanoma cell migration, growth, invasion and metastasis by suppressing the p38 MAPK and NF- $\kappa$ B pathway (12). Furthermore, a novel role of syntenin in regulating urothelial cell carcinoma (UCC) cell proliferation by epidermal growth factor receptor (EGFR) signaling has been observed, suggesting it is a promising target for developing detection, monitoring and therapeutic strategies for managing UCC (14). Although numerous studies on syntenin function in cancer have been performed, little is known regarding its roles and underlying molecular mechanism in the development of HCC.

In the present study, we investigated the expression of syntenin in HCC cell lines and explored its role in HCC cell proliferation and invasion. Furthermore, the underlying molecular mechanisms were also examined. 


\section{Materials and methods}

Antibodies and reagents. SB203580 (p38 MAPK inhibitor) was obtained from Sigma (St. Louis, MO, USA). Mouse anti-p38 and phospho-p38 antibodies were from Santa Cruz Biotechnology (Santa Cruz, CA, USA). Antibodies specific for syntenin (sc-100336) were purchased from Santa Cruz Biotechnology. Mouse anti-Ki67 monoclonal antibodies were from Sigma. The corresponding horseradish peroxidase-conjugated secondary antibodies were obtained from Calbiochem (La Jolla, CA, USA).

Cell culture. The human liver cancer cell lines HepG2, MHCC97-L, MHCC97-H and HCCLM3 were obtained from the American Type Culture Collection (ATCC; Rockville, MD, USA). Cells were maintained in Dulbecco's modified Eagle's medium (DMEM) containing 10\% fetal bovine serum, $100 \mu \mathrm{g} / \mathrm{ml}$ streptomycin and penicillin. Immortalized normal liver epithelial cells THLE3 were also purchased from ATCC and were maintained in bronchial epithelial growth medium, supplemented with $5 \mathrm{ng} / \mathrm{ml}$ epithelial growth factor, $70 \mathrm{ng} / \mathrm{ml}$ phosphoethanolamine and $10 \%$ fetal bovine serum. All cells were cultured in a humidified atmosphere at $37^{\circ} \mathrm{C}$ with $5 \%$ $\mathrm{CO}_{2}$.

Real-time PCR. Total RNA was isolated from human hepatoma cells HepG2 with the RNAiso Plus kit (Roche Diagnostics, Mannheim, Germany), and was then reverse-transcribed to synthesize first strand cDNA using the cDNA Synthesis kit (Fermentas). Then, $\sim 4 \mu \mathrm{l}$ cDNA was subjected to real-time PCR with specific primers for syntenin (sense, 5'-GAA TCC TGC AAA AAT GTC TC-3' and antisense, 5'-GCC ATG GTG CCG TGA ATT TTA-3'); EGFR (sense, 5'-TCG GGG AGC AGC GAT GCG AC-3' and antisense, 5'-TCA TGT GAT AAT TCA GCT C-3'); and MMP-2 (sense, 5'-CAG GGA GCG CTA CGA TGG A-3' and antisense, 5'-GAG CCA GGG CCA GCT CAG C-3'). Each reaction consisted of $10 \mu \mathrm{l} \mathrm{SYBR}{ }^{\circledR}$ Premix Ex Taq ${ }^{\mathrm{TM}}$ II, $10 \mu \mathrm{mol} / 1$ specific primers, $4 \mu \mathrm{l}$ of DNA and $\mathrm{H}_{2} \mathrm{O}$ to a final volume of $20 \mu \mathrm{l}$. The reaction conditions were introduced according to the instructions of SYBR Premix Ex Taq ${ }^{\mathrm{TM}}$ II kit (Takara Bio Inc., Otsu, Japan). For normalization, $\beta$-actin mRNA was used. All samples were performed in triplicate, and results were calculated according to the $2^{-\Delta \Delta C t}$ method.

Construction of syntenin expression vectors and stable transfection. To obtain the syntenin expression vectors, the syntenin cDNA was digested with BamHI and XhoI restriction enzymes, and was then ligated into the BamHI and Xhol cloning site of the pcDNA3.1(+) vector (Invitrogen) to induce syntenin expression. All sequences were confirmed by DNA sequencing. Transfection was performed using $8 \mu \mathrm{l}$ of Lipofectamine 2000 (Invitrogen) and $15 \mu \mathrm{g}$ of pcDNA3.1-syntenin or pcDNA3.1 empty vector in HCCLM3 cells. Forty-eight hours after transfection, $400 \mu \mathrm{g} / \mathrm{ml}$ of G418 was used to select the stable transfectants. The empty vector was packaged as a negative control. A limiting dilution was introduced to produce several stable syntenin-expressing clones.

RNA interference against EGFR. To knock down EGFR expression in HCCLM3 cells, the specific siRNA fragments of
EGFR and control siRNA were designed as previously described (15). The siRNA strands were synthesized by Shanghai Sangon Co., Ltd. (Shanghai, China). For transfection, HCCLM3 cells were seeded in 24-well microplates to reach $40-50 \%$ confluence. Then, cells were transfected with $2 \mu \mathrm{g} / \mathrm{ml}$ EGFR siRNA and $1 \mathrm{ml}$ Lipofectamine ${ }^{\mathrm{TM}}$ RNAi-MAX using the GeneSilencer ${ }^{\circledR}$ siRNA Transfection Reagent (GeneTherapy System, San Diego, CA, USA). Approximately $24 \mathrm{~h}$ later, the transfection efficiency was analyzed by western blotting as described above. Cell viability was evaluated by 3-(4,5-dimethyl-2-thiazolyl)-2,5-diphenyl-2H-tetrazolium bromide (MTT) assay.

Proliferation assays. Cell proliferation was assessed by MTT assay. Briefly, cells were seeded into 96-well culture plates at a density of $1 \times 10^{5}$ cells/well. After incubation for $24 \mathrm{~h}$, the culture medium was replaced with fresh medium containing $500 \mu \mathrm{g} / \mathrm{ml} \mathrm{MTT} \mathrm{(Sigma)} \mathrm{for} \mathrm{an} \mathrm{additional} 5 \mathrm{~h}$ at $37^{\circ} \mathrm{C}$ and $5 \%$ $\mathrm{CO}_{2}$. Then, the supernatant was replaced with $200 \mu \mathrm{l}$ isopropanol to dissolve formazan production and the absorbance was measured at $570 \mathrm{~nm}$ on a microplate reader (Bio-Rad, Hercules, CA, USA).

Colony formation assay. HCCLM3 cells transfected with syntenin were plated in $0.35 \%$ agar mixed culture medium in 12 -well plates. After 12 days, cells were washed with phosphate-buffered saline (PBS) three times, and were then fixed with methanol. Cell colony formation in soft agar was analyzed by counting the number of colonies under a stereoscopic microscope in triplicate of a 12 -well plate. The results are expressed as percentage control of colonies.

Invasion assay. The ability of cells to invade through Matrigelcoated filters was measured by a modified Boyden chamber (BD Biosciences, Bedford, MA, USA). Briefly, syntenin-overexpressed HCCLM3 cells were pre-treated with anti-MMP-2 antibody for $4 \mathrm{~h}$, and were then seeded at a density of $3.0 \times 10^{4}$ cells in the upper compartment. DMEM medium containing $10 \%$ fetal bovine serum was added to the lower compartment. After incubation for $48 \mathrm{~h}$ at $37^{\circ} \mathrm{C}$, cells were stained with hematoxylin and eosin (Sigma), and counted in six randomly selected high-powered fields in the center of each well. Each experiment was repeated three times.

Western blotting. Following rinses with PBS three times, cells were homogenized and lysed with RIPA lysis buffer (100 mM NaCl, 50 mM Tris- $\mathrm{HCl}$ pH 7.5, 1\% Triton X-100, $1 \mathrm{mM}$ EDTA, $10 \mathrm{mM}$ b-glycerophosphate, $2 \mathrm{mM}$ sodium vanadate and protease inhibitor) to extract the total protein, and then a BCA assay (Pierce, Rockford, IL, USA) was introduced to quantify the protein concentrations. Following electrophoresis by SDS-PAGE, the obtained protein was transferred onto a polyvinylidene difluoride (PVDF) membrane in a semi-dry transblot apparatus. To perform the western blot assay, non-specific binding was blocked by incubating with $5 \%$ non-fat milk in TBST buffer at room temperature at $4^{\circ} \mathrm{C}$ overnight. Then, immune-detection of syntenin, EGFR, Ki67, MMP-2, p-p38 MAPK and p38 MAPK was performed using specific antibodies against them for $1 \mathrm{~h}$ at $37^{\circ} \mathrm{C}$, followed by incubation with HRP-conjugated secondary antibodies for 

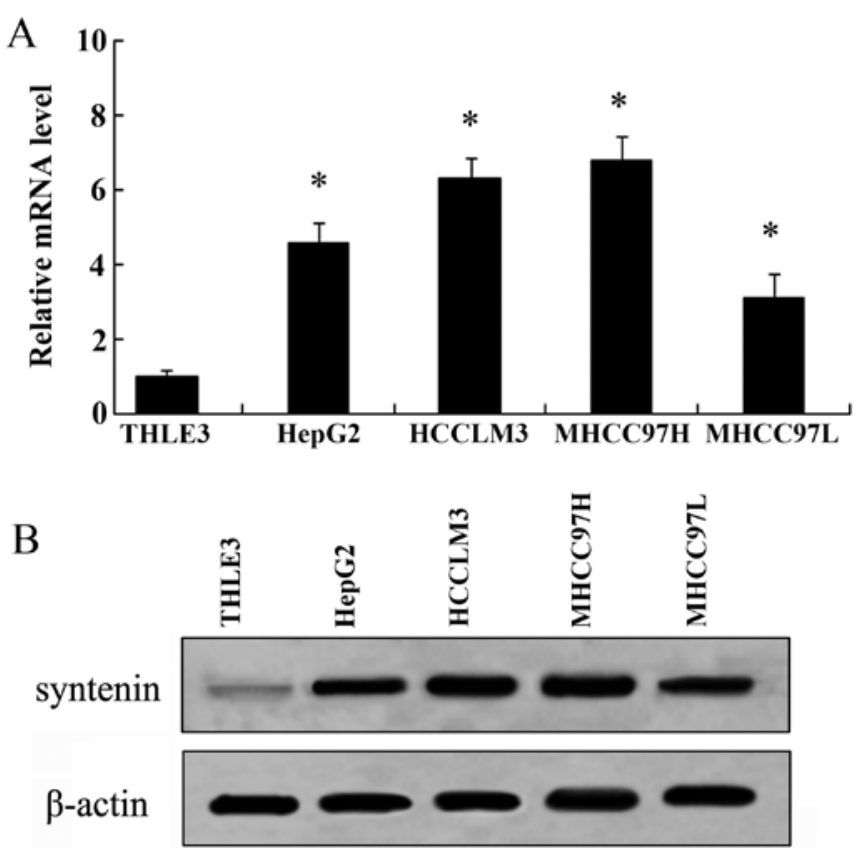

Figure 1. Expression of syntenin in human hepatoma cell lines. To determine the expression levels of syntenin in hepatoma cells, four HCC cell lines, HepG2, MHCC97-L, MHCC97-H and HCCLM3, and normal liver epithelial cells THLE3 were used. (A) The mRNA levels of syntenin in these cells were analyzed by RT-PCR. (B) The corresponding syntenin protein levels were evaluated by western blotting. " $\mathrm{P}<0.05$ vs. THLE3 liver epithelial cells.

$1 \mathrm{~h}$. To visualize the bound antibodies, the LumiGLO reagent (KPL, Gaithersburg, MD, USA) was used. The protein expression levels were normalized by $\beta$-actin.

Statistical analysis. The Student's t-test was used to evaluate statistically significant differences between two groups in all relevant experiments. All numerical results were analyzed by SPSS 11.0 and are presented as means \pm SD. A P-value $<0.05$ was considered to indicate a statistically significant result.

\section{Results}

Expression of syntenin is increased in HCC cell lines. Syntenin has been confirmed to be upregulated in several cancers and is critical for the development of cancer $(9,13)$. However, research regarding syntenin in HCC remains to be performed. To address this, we analyzed the expression of syntenin in various HCC cell lines. Compared with normal liver epithelial cells THLE3, an apparent increase in syntenin mRNA levels was observed in all four HCC cell lines including HepG2, MHCC97-L, MHCC97-H and HCCLM3 by RT-PCR analysis (Fig. 1A). Furthermore, western blot analysis demonstrated that the expression levels of syntenin were also markedly upregulated in these four HCC cell lines, compared with THLE3 cells (Fig. 1B). Moreover, the mRNA and protein levels of syntenin in the high metastatic potential cell line MHCC97-H were higher than in the low-invasive cell line MHCC97-L, indicating a potential correlation between syntenin expression and the metastatic ability of HCC cell lines. Collectively, these results corroborated that syntenin is upregulated in HCC cells, particularly in higher metastatic cells.
A

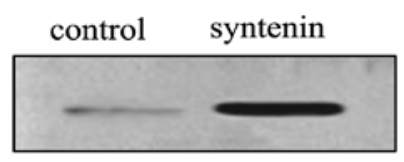

syntenin

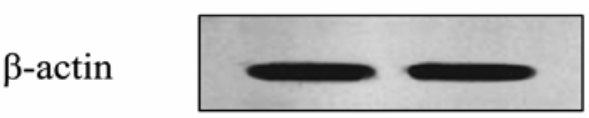

B

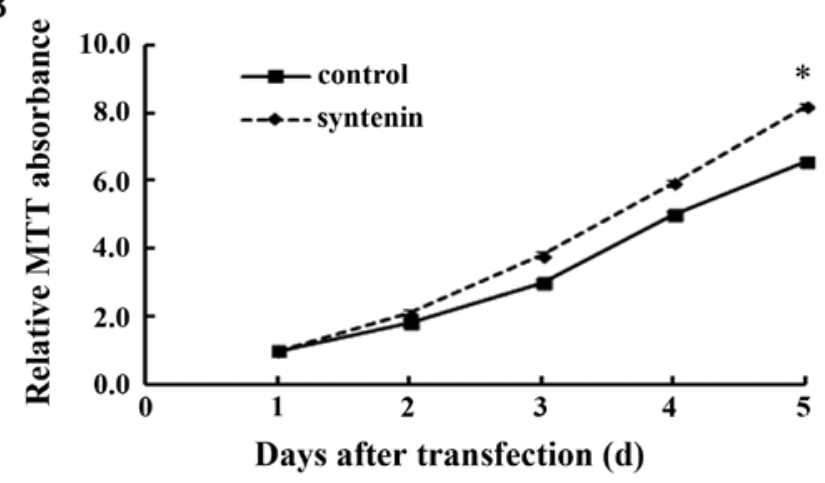

$\mathrm{C}$ control

syntenin
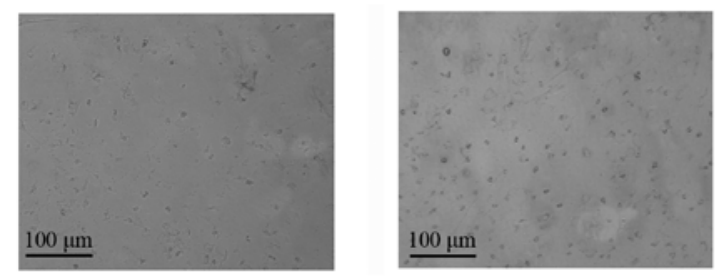

है

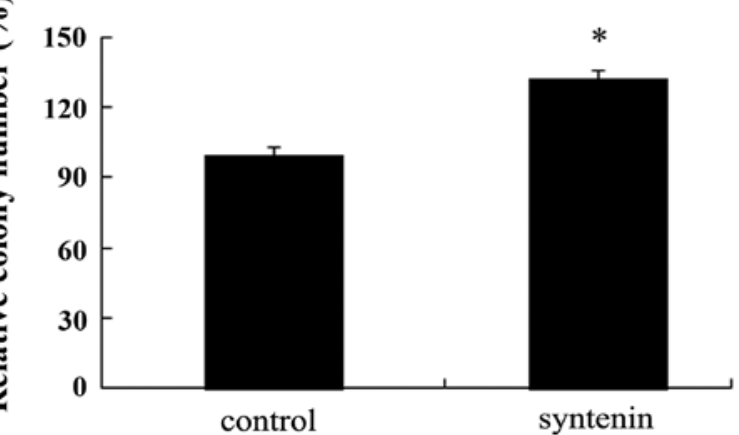

Figure 2. Syntenin overexpression enhances hepatoma cell proliferation and colony formation. After transfection of recombinant pcDNA3.1-syntenin and empty vector into HCCLM3 cells, the expression level of syntenin protein was observed (A) and termed HCCLM-150. Cells transfected with empty vector were used as the control group. When cells overexpressed syntenin in HCCLM-150, the proliferation ability of cells was analyzed by MTT assay (B). The colonies were observed in soft agar (C). ${ }^{*} \mathrm{P}<0.05$.

Overexpression of syntenin enhances HCCLM3 cell proliferation in vitro. To clarify the role of syntenin in the development and progression of $\mathrm{HCC}$, we assessed the effect of syntenin overexpression on HCCLM3 cell growth. To quantify the direct contribution of syntenin in hepatoma, the recombinant pcDNA3.1-syntenin was transfected into HCCLM3 cells, and then a stable syntenin overexpression clone termed HCCLM3-150 cell line was obtained. As shown in Fig. 2A, a striking upregulation of syntenin protein was detected in the HCCLM3-150 cell line, compared with the vector control clone. Based on the stable expression of syntenin in HCCLM3-150 cells, we evaluated syntenin function on cell growth by MTT 


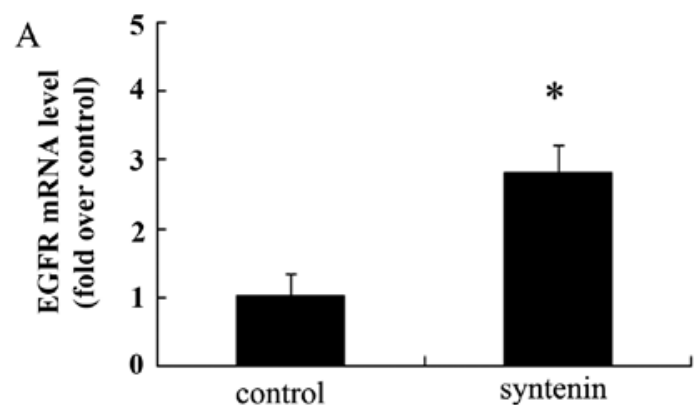

B

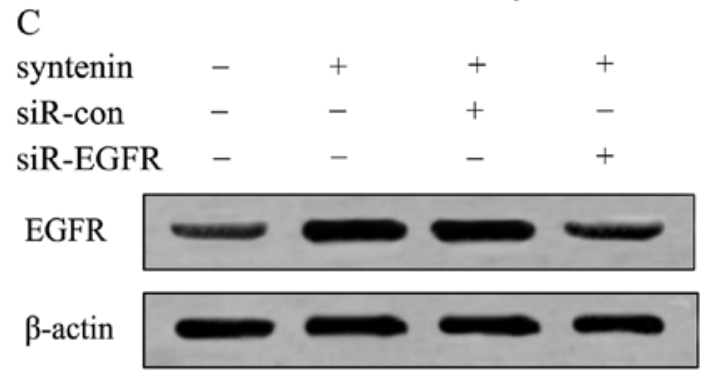

D

EGFR

control syntenin

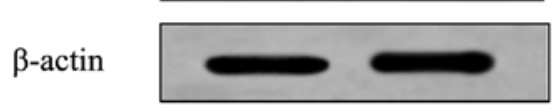

E

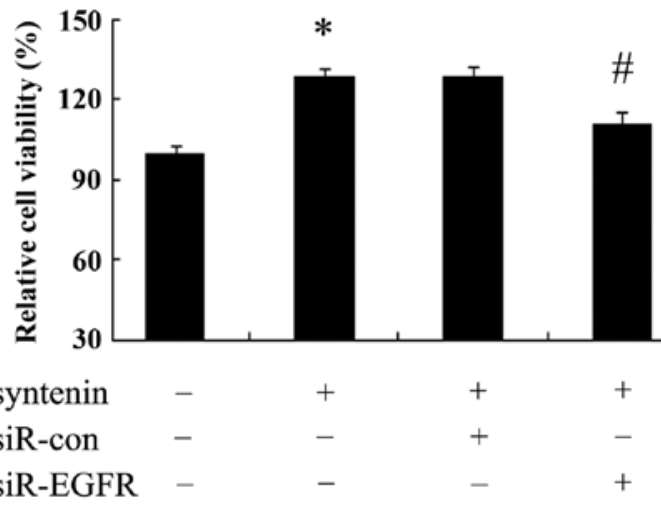

ki67

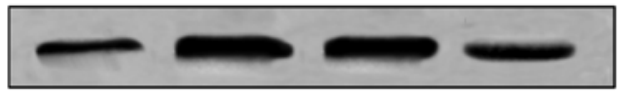

$\beta$-actin

syntenin

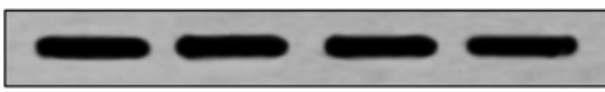

siR-con

$-$

$+$

$+$

$+$

siR-EGFR

Figure 3. Syntenin promotes hepatoma cell proliferation in an EGFR-dependent manner. When HCCLM-150 stably overexpressed syntenin, the expression levels of EGFR mRNA (A) and protein (B) were examined by RT-PCR and western blotting. After blocking EGFR expression with its specific siRNA, the silencing effect of EGFR was detected in HCCLM-150 cells (C). Then, cell proliferation was corroborated by MTT assay in EGFR-silenced HCCLM-150 cells (D). The expression levels of ki67 were also analyzed by western blotting (E). ${ }^{*} \mathrm{P}<0.05$ vs. control group. ${ }^{\#} \mathrm{P}<0.05$ vs. siR-con group.

and colony formation assays. As shown in Fig. 2B, an apparent increase in cell proliferation was observed as the gradually increased transfection times in syntenin-overexpressed group, compared with the control group. Furthermore, syntenin overexpression displayed more colonies in contrast to controls (Fig. 2C). Therefore, these results showed that syntenin transfection strongly promotes cell proliferation.

Syntenin enhances hepatoma cell proliferation in an EGFRdependent manner. It is generally believed that EGFR is overexpressed in several cancers, including colorectal cancer, HCC and urothelial carcinoma, and can activate a cascade of multiple signaling pathways that facilitate the tumor growth process (16-18). The fact that syntenin can promote hepatoma cell proliferation was demonstrated above. However, the precise molecular mechanism of this action remains unclear. Hence, we attempted to link EGFR to syntenin-induced cell proliferation mechanistically. When cells were stably transfected with syntenin, the mRNA levels of EGFR were 2.7-fold over controls, indicating that elevated syntenin expression strongly upregulated EGFR mRNA levels (Fig. 3A). Moreover, a similar upregulation of EGFR protein levels was also ascertained by western blot assay (Fig. 3B). Further mechanistic analysis corroborated that EGFR silencing significantly reduced cell viability induced by syntenin overexpression (Fig. 3C). Consistently, the expression levels of proliferation marker Ki67 were markedly attenuated when blocking EGFR expression in HCCLM3-150 cells (Fig. 3D). Taken together, our data demonstrated that syntenin overexpression promotes hepatoma cell proliferation predominantly through the EGFR pathway.

Elevated syntenin expression stimulates hepatoma cell invasion by MMP-2. To further assess the function of syntenin in hepatoma cells, we detected cell invasion ability in HCCLM-150, which stably overexpressed syntenin. As shown in Fig. 4, the forced syntenin expression notably upregulated the number of invaded cells from 56 to 135 compared to the control groups, indicating it is a positive regulator for HCC cell invasion. It is known that MMP-2 can degrade extracellular matrix to facilitate tumor cell invasion (19). Thus, we linked the MMP-2 to syntenin-induced cell invasion. Following transfection of syntenin in HCCLM3 cells, the expression levels of MMP-2 mRNA were clearly increased and were 3.75-fold 


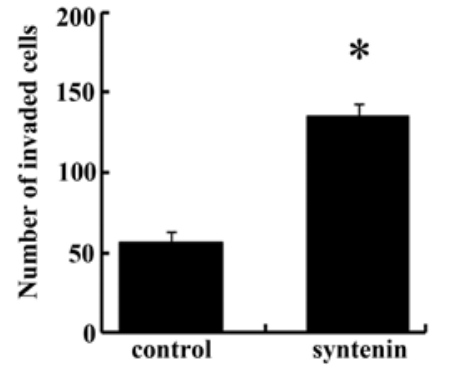

Figure 4. Elevated syntenin expression induces hepatoma cell invasion. Following transfection with pcDNA3.1-syntenin and empty vector into HCCLM3 cells, cell invasion ability was examined by Transwell analysis.

\section{A}

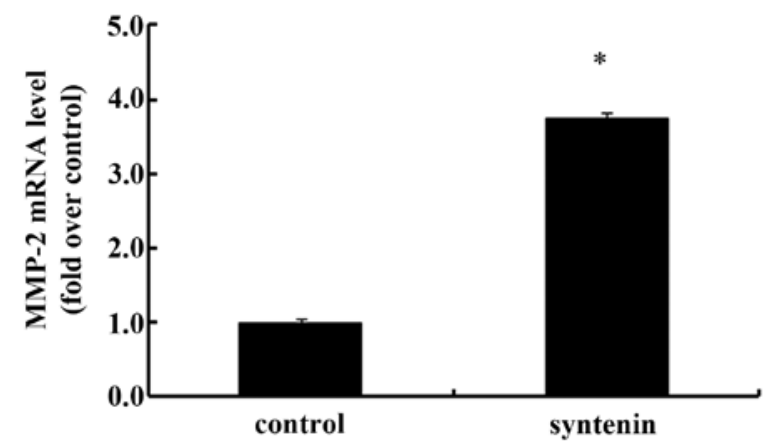

B

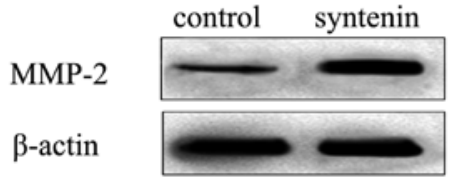

C

Anti-MMP- $2 \quad-\quad+$

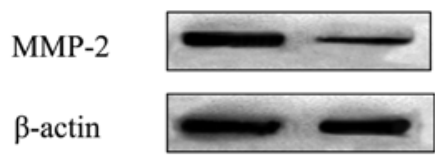

$\mathrm{D}$

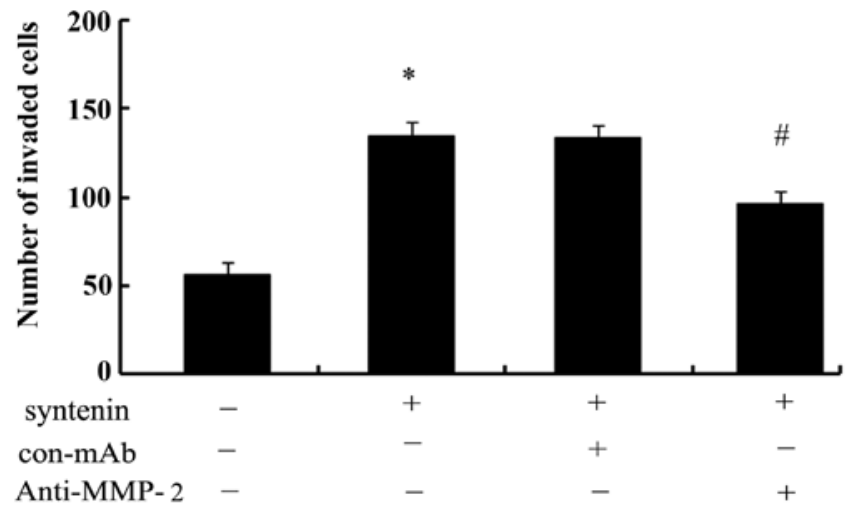

Figure 5. MMP-2 is responsible for the syntenin-triggered cell invasion. The expression levels of MMP-2 mRNA and protein were examined by RT-PCR (A) and western blotting (B) in cells, which overexpressed syntenin or not. After preconditioning with anti-MMP-2 antibody, the expression level of MMP-2 was confirmed by western blotting in HCCLM-150 cells (C). The corresponding effect of MMP-2 on syntenin-induced cell invasion was assessed by Transwell assay. ${ }^{*} \mathrm{P}<0.05 .{ }^{\#} \mathrm{P}<0.05$ vs. control antibody-treated group.

times higher than those of the controls (Fig. 5A). Consistent with the above observation, syntenin overexpression significantly enhanced the expression of MMP-2 protein (Fig. 5B).
A

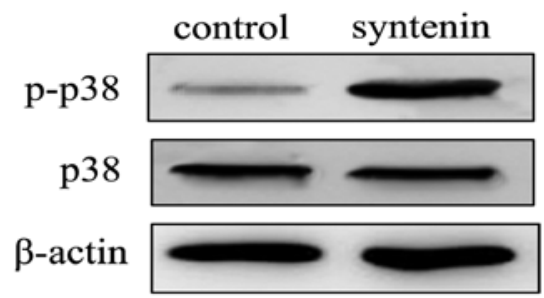

B
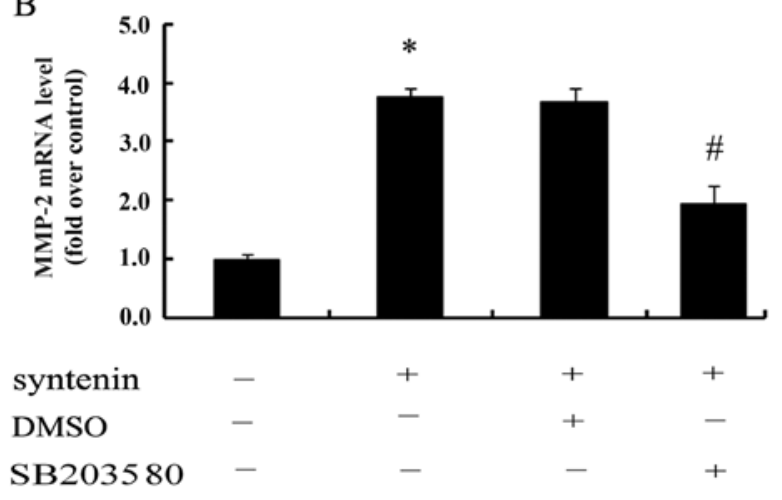

$\mathrm{C}$
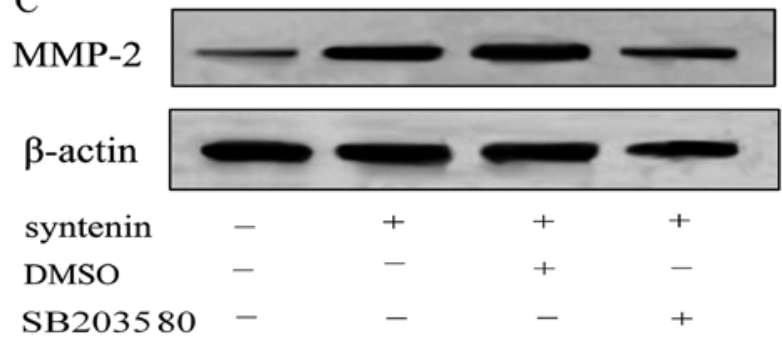

Figure 6. Syntenin overexpression induces MMP-2 expression by the p38 MAPK pathway. The phosphorylation of p38 MAPK was detected in syntenin-transfected or not groups (A). Following pretreatment with or without the p38 MAPK inhibitor SB203580 (25 $\mu \mathrm{M})$ for $1 \mathrm{~h}$, the MMP-2 mRNA levels (B) and protein levels (C) were analyzed in HLCCM-150 cells. ${ }^{*} \mathrm{P}<0.05$. ${ }^{\text {}} \mathrm{P}<0.05$ DMSO-treated group.

To further analyze the underlying mechanism involved in syntenin-induced cell invasion, we silenced the expression levels of MMP-2 with its specific antibody and a notable downregulation of MMP-2 levels was observed in HCCLM3150 cells (Fig. 5C). Also, MMP-2 silencing clearly abrogated syntenin-induced cell invasion number (Fig. 5D). Hence, these data confirmed that syntenin enhances cell invasion ability in an MMP-2-dependent manner.

Syntenin-induced MMP-2 expression is regulated by the p38 MAPK pathway. To further clarify the mechanism associated with syntenin-triggered hepatoma cell invasion through MMP-2, we evaluated the activation of p38 MAPK. Only slight phosphor-p38 MAPK was observed in the control group; however, when cells overexpressed syntenin, the expression of p-p38 MAPK was significantly increased, suggesting that syntenin overexpression induced the activation of the $\mathrm{p} 38$ MAPK pathway (Fig. 6A). Notably, the specific inhibitor of p38 MAPK, SB203580, strikingly blocked syntenin-induced MMP-2 mRNA levels (Fig. 6B), concomitant with the reduction of MMP-2 protein levels (Fig. 6C). These results revealed that syntenin promotes cell invasion via MMP-2 expression through the activation of the p38 MAPK pathway. 


\section{Discussion}

Hepatocellular carcinoma (HCC) is the sixth most common malignancy, resulting in more than 560,000 deaths per year, and it is the third leading cause of tumor-related mortality around the world $(1,2,20)$. The present study demonstrated that syntenin was markedly upregulated in several HCC cell lines compared with its expression in normal liver epithelial THLE3 cells. Moreover, syntenin upregulation promoted HCCLM3 cell proliferation by epidermal growth factor receptor (EGFR) signaling. Notably, syntenin overexpression enhanced hepatoma cell invasion through the activation of the p38 MAPK pathway. These findings suggest that syntenin may play an important role in the development and progression of hepatoma.

Syntenin is also known as mda- 9 and was originally cloned from human melanoma cells. As a PDZ domain-containing molecule, syntenin can exert multiple roles and regulate diverse and central physiologic processes $(8,9)$. The function of syntenin in cancer has attracted increasing attention for its important roles in the development of various cancers, including melanoma, breast and bladder cancer $(10,12,13)$. It has been confirmed that syntenin is highly expressed in several cancer cells and tissues, and it is involved in the progression of cancer, including cancer cell growth, invasion and metastasis $(9,21,22)$. However, its function in the development of hepatoma remains unclear. In the present study, we firstly confirmed a similar high expression of syntenin mRNA and protein levels in HCC cells. Moreover, cell proliferation ability and clone formation ability were strongly elevated after stable expression of syntenin in HCCLM3 cells, indicating a critical role of syntenin in hepatoma cell growth. However, the underlying mechanism remains undefined.

EGFR belongs to the ErbB proto-oncogene receptors family (ErbB tyrosine kinase receptors), and its cognate ligand has been considered as a common component of various cancer types to induce tumor growth $(23,24)$. EGFR overexpression has been confirmed in multiple carcinomas including breast cancer, urothelial cell carcinoma and hepatoma $(10,16)$. It is believed that EGFR can trigger a cascade of multiple signaling pathways to regulate cancer cell proliferation, maintenance, invasion, metastasis, survival and prognosis $(16,17,25,26)$. Furthermore, EGFR elicits a vital role in MDA-9/ syntenin-induced urothelial carcinoma cell proliferation (14). Accordingly, to further corroborate the molecular mechanism underlying syntenin-induced HCC cell proliferation, we analyzed the expression levels of EGFR. When syntenin was stably transfected into HCCLM cells, the expression levels of EGFR mRNA and protein were notably upregulated. More importantly, blocking its expression with specific siRNA markedly attenuated syntenin-triggered cell proliferation as well as the expression of proliferation marker Ki67, suggesting a pivotal function of EGFR in syntenin-regulated hepatoma cell proliferation.

The high invasiveness is critical for tumor progression and is known to be a vital contributor to morbidity and mortality in cancer (27). The fact that a higher expression of syntenin was observed in the high metastatic potential cell line MHCC97-H, compared with the low-invasive cell line MHCC97-L, indicated a potential role in HCC cell invasion and metastasis. To better understand the roles of syntenin in the development of hepatoma, we further assessed the effect of syntenin overexpression on cell invasion. As expected, syntenin upregulation significantly enhanced hepatoma cell invasion. Matrix metalloproteinases (MMPs) are known to possess an indispensable role during the degradation of extracellular matrix (ECM), a crucial step in tumor invasion and metastasis $(28,29)$. Among them, MMP-2 has exhibited an important role in hepatoma cell adhesion and invasion $(30,31)$. To clarify the underlying mechanism involved in syntenin-induced cell invasion, we well linked MMP-2 together. In the present study, syntenin overexpression enhanced the expression levels of MMP- 2 mRNA and protein. When silencing its expression with anti-MMP-2 antibody, syntenin-triggered hepatoma cell invasion ability was clearly abrogated, indicating that syntenin may enhance cell invasion by MMP-2 expression.

P38 MAPK pathways can trigger a cascade of responses, from cell growth and proliferation to motility and invasion, which drive tumor development and progression $(32,33)$. To further elucidate the exact molecular mechanism involved in syntenin-induced cancer cell invasion via MMP-2 expression, the phosphor-p38 MAPK was explored. After overexpression of syntenin, the expression levels of p-p38 MAPK were significantly enhanced, indicating a pivotal effect of syntenin on the activation of the p38 MAPK pathway. Following pretreatment with SB203580, syntenin-induced MMP-2 expression levels were significantly reduced. Accordingly, we can conclude that syntenin may promote the invasiveness of hepatoma cells by MMP-2 expression through the p38 MAPK pathway.

In conclusion, we confirmed the upregulation of syntenin in hepatoma cells. In the present study, syntenin overexpression enhanced hepatoma cell proliferation and invasion by EGFR pathway and p38 MAPK signaling, which ultimately benefit hepatoma development and progression. These findings provide insight into how syntenin accelerates the pathogenesis of hepatoma. Therefore, syntenin may prove to be a promising therapeutic agent against hepatoma. Further studies will focus on its function and regulation mechanism on hepatoma progression in vivo. Furthermore, its PDZ domain function in the development of hepatoma will also be investigated in a forthcoming study.

\section{Acknowledgements}

Financial support was provided by the National Natural Science Foundation of China (NSFC) (no. 81101873).

\section{References}

1. Jelic S and Sotiropoulos GC; ESMO Guidelines Working Group: Hepatocellular carcinoma: ESMO Clinical Practice Guidelines for diagnosis, treatment and follow-up. Ann Oncol 21: v59-v64, 2010.

2. Yang JD and Roberts LR: Hepatocellular carcinoma: a global view. Nat Rev Gastroenterol Hepatol 7: 448-458, 2010.

3. McGlynn KA and London WT: International liver cancer incidence trends - letter. Cancer Epidemiol Biomarkers Prev 21: 384-385, 2012.

4. Venook AP, Papandreou C, Furuse J and de Guevara LL: The incidence and epidemiology of hepatocellular carcinoma: a global and regional perspective. Oncologist 15: 5-13, 2010.

5. Altekruse SF, McGlynn KA and Reichman ME: Hepatocellular carcinoma incidence, mortality, and survival trends in the United States from 1975 to 2005. J Clin Oncol 27: 1485-1491, 2009. 
6. Sarkar D, Boukerche H, Su Z-Z and Fisher PB: $m d a-9 /$ syntenin: recent insights into a novel cell signaling and metastasis-associated gene. Pharmacol Ther 104: 101-115, 2004.

7. Das SK, Bhutia SK, Sokhi UK, et al: Raf kinase inhibitor RKIP inhibits MDA-9/syntenin-mediated metastasis in melanoma Cancer Res 72: 6217-6226, 2012.

8. Yu Y and Schachner M: Syntenin-a promotes spinal cord regeneration following injury in adult zebrafish. Eur J Neurosci 38: 2280-2289, 2013.

9. Gangemi R, Mirisola V, Barisione G, et al: Mda-9/syntenin is expressed in uveal melanoma and correlates with metastatic progression. PLoS One 7: e29989, 2012.

10. Yang Y, Hong Q, Shi P, Liu Z, Luo J and Shao Z: Elevated expression of syntenin in breast cancer is correlated with lymph node metastasis and poor patient survival. Breast Cancer Res 15: R50, 2013.

11. Das SK, Bhutia SK, Kegelman TP, et al: MDA-9/syntenin: a positive gatekeeper of melanoma metastasis. Front Biosci 17: $1-15,2012$.

12. Boukerche H, Su ZZ, Prévot C, Sarkar D and Fisher PB: mda-9/ syntenin promotes metastasis in human melanoma cells by activating c-Src. Proc Natl Acad Sci 105: 15914-15919, 2008.

13. Koo TH, Lee JJ, Kim EM, Kim KW, Kim HD and Lee JH: Syntenin is overexpressed and promotes cell migration in metastatic human breast and gastric cancer cell lines. Oncogene 21 : 4080-4088, 2002.

14. Dasgupta S, Menezes ME, Das SK, et al: Novel role of MDA-9/ syntenin in regulating urothelial cell proliferation by modulating EGFR signaling. Clin Cancer Res 19: 4621-4633, 2013.

15. Han W, Pan H, Chen Y, et al: EGFR tyrosine kinase inhibitors activate autophagy as a cytoprotective response in human lung cancer cells. PLoS One 6: e18691, 2011.

16. Spano J, Fagard R, Soria JC, Rixe O, Khayat D and Milano G: Epidermal growth factor receptor signaling in colorectal cancer: preclinical data and therapeutic perspectives. Ann Oncol 16: 189-194, 2005.

17. Kannangai R, Sahin F and Torbenson MS: EGFR is phosphorylated at Ty845 in hepatocellular carcinoma. Mod Pathol 19: 1456-1461, 2006.

18. Mitsudomi T and Yatabe Y: Epidermal growth factor receptor in relation to tumor development: EGFR gene and cancer. FEBS J 277: 301-308, 2010.

19. Wang X, Lu N, Niu B, Chen X, Xie J and Cheng N: Overexpression of Aurora-A enhances invasion and matrix metalloproteinase-2 expression in esophageal squamous cell carcinoma cells. Mol Cancer Res 10: 588-596, 2012.
20. Kim H and Lim HY: Novel EGFR-TK inhibitor EKB-569 inhibits hepatocellular carcinoma cell proliferation by AKT and MAPK pathways. J Korean Med Sci 26: 1563-1568, 2011.

21. Boukerche $\mathrm{H}$, Aissaoui $\mathrm{H}$, Prévost $\mathrm{C}$, et al: OC-13 enhanced expression of Mda-9/syntenin induced through the tissue-factor pathway in melanoma promotes tumor cell migration and invasion. Thromb Res 125 (Suppl 2): S164, 2010.

22. Kim WY, Jang JY, Jeon YK, Chung DH, Kim YG and Kim CW: Syntenin increases the invasiveness of small cell lung cancer cells by activating p38, AKT, focal adhesion kinase and SP1. Exp Mol Med 46: e90, 2014.

23. Bazley LA and Gullick WJ: The epidermal growth factor receptor family. Endocr Relat Cancer 12 (Suppl 12): S17-S27, 2005.

24. da Cunha Santos G, Shepherd FA and Tsao MS: EGFR mutations and lung cancer. Annu Rev Pathol 6: 49-69, 2011.

25. Hirsch FR, Varella-Garcia M and Cappuzzo F: Predictive value of EGFR and HER2 overexpression in advanced non-small-cell lung cancer. Oncogene 28: S32-S37, 2009.

26. Nicholson RI, Gee JM and Harper ME: EGFR and cancer prognosis. Eur J Cancer 37: 9-15, 2001.

27. Duffy MJ, McGowan PM and Gallagher WM: Cancer invasion and metastasis: changing views. J Pathol 214: 283-293, 2008.

28. Kessenbrock K, Plaks V and Werb Z: Matrix metalloproteinases: regulators of the tumor microenvironment. Cell 141: 52-67, 2010.

29. Gialeli C, Theocharis AD and Karamanos NK: Roles of matrix metalloproteinases in cancer progression and their pharmacological targeting. FEBS J 278: 16-27, 2011.

30. Lara-Pezzi E, Gómez-Gaviro MV, Gálvez BG, et al: The hepatitis B virus $X$ protein promotes tumor cell invasion by inducing membrane-type matrix metalloproteinase- 1 and cyclooxygenase-2 expression. J Clin Invest 110: 1831-1838, 2002.

31. Giannelli G, Bergamini C, Marinosci F, et al: Clinical role of MMP-2/TIMP-2 imbalance in hepatocellular carcinoma. Int J Cancer 97: 425-431, 2002.

32. Wagner EF and Nebreda AR: Signal integration by JNK and p38 MAPK pathways in cancer development. Nat Rev Cancer 9: 537-549, 2009.

33. del Barco Barrantes I and Nebreda A: Roles of p38 MAPKs in invasion and metastasis. Biochem Soc Trans 40: 79-84, 2012 